\title{
REVISIÓN \\ Proteínas transmembranales de organismos tipo rickettsia (OTR) en animales acuáticos: Factores de adherencia, invasión e infección
}

\author{
Transmembrane proteins rickettsia-like organisms (RLO) in aquatic animals: \\ Adhesion, invasion and infection factors \\ Jesús Pérez-Acosta ${ }^{1}$, Marcel Martínez-Porchas ${ }^{2}$, Teresa Gollas-Galván ${ }^{2 *}$, \\ Luis R. Martínez-Córdova ${ }^{1}$, Luis E. Gutiérrez-Millán ${ }^{1}$ \\ y Marco López-Torres ${ }^{1}$
}

${ }^{1}$ Departamento de Investigaciones Científicas y Tecnológicas de la Universidad de Sonora, Hermosillo, Sonora, México
${ }^{2}$ Centro de Investigación en Alimentación y Desarrollo, A.C. Hermosillo, Sonora, México. *tgollas@ciad.mx

\begin{abstract}
Besides participating in the transport of essential compounds and as recipients of phages, transmembrane proteins of rickettsia-like organisms (RLO), play an important role in the infection process of commercially important organisms such as fish, mollusk and crustaceans. Recently a new classification of RLO which divides them into four groups was reported: ancestral, typhus, the spotted fever and transitional. To date, only the typhus (TG) and spotted fever (SFG) groups have been reported as pathogenic of marine organisms. The outer membrane surface of the TG group has antigens type 0, 1, 2, 4, 5 (Sca0, Sca1, Sca2, Sca4, $\mathrm{Sca} 5)$. Sca0 are also known as outer membrane proteins $\mathrm{A}(\mathrm{OmpA})$ and Sca5 as outer membrane proteins B (OmpB). Other outer membrane proteins such as enzymes called translocase (TIC) have been detected, including translocase 1, 4 and 5 (Tlc1, Tlc4 and TIc5). All these proteins (except for Sca0) have been detected in the SFG group. These proteins vary in features and functions. However, the information of RLO affecting marine organisms is not as common as that of terrestrial mammals. An analysis of the state of the art on the study of transmembrane proteins of RLO in marine organisms is performed in this review; additionally, the hypothetical infection mechanism of these RLO is inferred by using this information together with that reported of RLO affecting terrestrial organisms.
\end{abstract}

Key words: Rickettsia-like, RLO, TG, SFG, rOmpA, rOmpB, Tlc, Sca

\begin{abstract}
Resumen.- Las proteínas transmembranales de organismos tipo rickettsia (OTR) además de participar en el transporte de compuestos esenciales y como receptoras de fagos, desempeñan un papel importante en el proceso de infección sobre organismos de importancia comercial como peces, moluscos y crustáceos. Recientemente se dio a conocer una nueva clasificación de los OTR la cual los divide en cuatro grupos: ancestral, tifo, de la fiebre manchada y transicional. Hasta el momento, solo los grupos tifo y de la fiebre manchada (TG y SFG, por sus siglas en inglés 'Typhus Group' y 'Spotted Fever Group') han sido reportados como patogénicos de organismos acuáticos. El grupo TG posee en su membrana externa antígenos celulares de superficie tipo 0 , 1, 2, 4, 5 (Sca0, Sca1, Sca2, Sca4, Sca5, por sus siglas en inglés Surface cell antigens). Sca0 también es conocido como proteína transmembranal A y Sca5 como proteína transmembranal B (OmpA y OmpB, por sus siglas en inglés 'Outer Membrane Protein'). Además se han encontrado en esta membrana externa algunas enzimas Ilamadas translocasas (Tlc) entre las cuales se encuentran la traslocasa 1, 4 y 5 (Tlc1, Tlc4 y Tlc5). En el grupo SFG también se han detectado estas proteínas con excepción de Sca0. Estas proteínas varían en cuanto a características y funciones. Sin embargo, la información de OTR que afectan organismos acuáticos, no es tan común como en mamíferos terrestres. En esta revisión se hace un análisis del estado del arte del estudio de proteínas transmembranales de OTR en organismos acuáticos y se contrasta con la información reportada respecto a OTR de origen terrestre, con el objetivo de inferir el posible mecanismo de infección de este tipo de patógenos.
\end{abstract}

Palabras clave: Rickettsias, OTR, TG, SFG, OmpA, OmpB, Tlc, Sca

\section{INTRODUCCIÓN}

Los organismos tipo rickettsia (OTR) son bacterias intracelulares obligadas, $\alpha$-proteobacterias, Gram negativas (Gram-) y se multiplican exclusivamente dentro de las células del organismo hospedero (Ferrantini et al. 2009). Los OTR son organismos pleomórficos, cuya forma predominante es de bacilos, teniendo un segundo fenotipo de estructura helicoidal con 8 flagelos en su vértice basal (Morales-Covarrubias et al. 2011, Oliver et al. 2015). Recientemente los OTR han sido clasificados en cuatro grupos: ancestral, tifo(TG), de la fiebre manchada (SFG) y transicional (Gillespie et al. 2008). La ocurrencia de estos 
patógenos OTR se ha incrementado en los últimos años en la industria acuícola y pesquerías de mar abierto (Oliver et al. 2015).

Se han encontrado diversas especies de moluscos, peces y crustáceos que han sido infectadas por OTR. El modo de infección en animales acuáticos se puede dar por la colonización de las superficies branquiales e intestinales, para posteriormente ingresar a las células en los órganos blancos. La infección por la ruta gástrica es un punto clave en la interacción de algunos tipos de OTR, como lo es la bacteria de la necrosis hepatopancreática (BNHP), sirviendo como vía de colonización, donde interactúan con las células hepatopancreáticas
E, R, F y B, y provocan daño en los túbulos, perdida de vacuolas lipídicas, granulación e infiltración hemocítica en el hepatopáncreas (Morales-Covarrubias et al. 2011).

La primera infección de OTR reportada en moluscos se registró en la almeja Mya arenaria en la Bahía Chesapeake Estados Unidos, en 1976 (Harshbarger et al. 1977); desde entonces se han reportado más de 30 especies de moluscos infectados por este tipo de patógeno (Tabla 1). Las principales células que se ven afectadas en los moluscos son del hepatopáncreas y manto, aunque también se pueden observar lesiones en la glándula digestiva, branquias y tejido conectivo (Zhu \& Wu 2008).

Tabla 1. Especies de moluscos infectados con organismos tipo rickettsia (OTR). Obtenida de los reportes de Hines \& Diggles 2002, Azevedo et al. (2005, 2006); Ceuta \& Boehs (2012), Zewen et al. (2012) y Gollas-Galván et al. (2013) / Mollusk species infected with rickettsia-like organisms (RLO). Obtained from the reports of Hines \& Diggles 2002, Azevedo et al. (2005, 2006); Ceuta \& Boehs (2012), Zewen et al. (2012) and Gollas-Galván et al. (2013)

\begin{tabular}{lll}
\hline $\begin{array}{l}\text { Familias hospederas } \\
\text { de OTR }\end{array}$ & \multicolumn{1}{c}{ Nombre común } & \multicolumn{1}{c}{ Especie } \\
& & \\
\hline Arcidae & Almeja berberecho sangre & Tegillarca granosa \\
Cariidae & Berberecho común & Cerastoderma edule \\
Cultellidae & Almeja navaja del Pacífico & Siliqua patula \\
Donacidae & Coquina & Donax trunculus \\
Haliotidae & Oreja de mar & Haliotis tuberculate \\
Mydiae & Almeja de suave cáscara & Mya arenaria \\
Mytilidae & Mejillón fanguero de Guayana & Mytella guyanensis \\
Mytilidae & Mejillón de California & Mytilus californianus \\
Mytilidae & Mejillón azul & Mytilus edulis \\
Mytilidae & Mejillón del mediterráneo & Mytilus galloprovincialis \\
Ostreidae & Ostra portuguesa & Crassostrea angulate \\
Ostreidae & Ostra china & Crassostrea ariakensis \\
Ostreidae & Ostra del Pacífico & Crassostrea gigas \\
Ostreidae & Ostra de mangle ahuecada & Crassostrea rizophorae \\
Ostreidae & Ostra oriental & Crassostrea virginica \\
Ostreidae & Ostra plana europea & Ostrea edulis \\
Pectinidae & Escalope de bahía & Argopecten irradiens \\
Pectinidae & Escalope jaspeado & Chlamys varia \\
Pectinidae & Escalope reina & Chlamys opercularis \\
Pectinidae & Escalope japonés & Patinopecten yessoensis \\
Pectinidae & Escalope del mar del Atlántico & Placopecten magellanicus \\
Pectinidae & Escalope rey & Pecten maximus \\
Pectinidae & Escalope de Nueva Zelanda & Pecten novaezelandiae \\
Pteriidae & Ostra perla & Pinctada máxima \\
Tellinidae & Tellin Delgado & Tellina tenuis \\
Tridacnidae & Almeja madriguera & Tridacna crocea \\
Veneridae & Quahog del norte & Mercenaria mercenaria \\
Veneridae & Almeja manila & Ruditapes philippinarum \\
Veneridae & Almeja concha de alfombra & Ruditapes decussatus \\
Veneridae & Almeja japonesa de cuello corto & Tapes japonica \\
Veneridae & Almeja concha de alfombra & Tapes pullastra \\
\hline & & \\
\hline & &
\end{tabular}


Respecto a los crustáceos afectados por OTR, los casos reportados con esta enfermedad no han aumentado significativamente hasta el 2016 y solo se cuenta con poco más de 18 especies (Tabla 2). Las zonas en las cuales se ha observado una mayor afección causada por los OTR en crustáceos son los túbulos hepatopancreáticos, tejido, músculo, intestino, branquias y hemolinfa. También se denota un menor tamaño de los organismos infectados (Wang et al. 2011).

En camarones y otros crustáceos infectados por OTR los signos clínicos observables aparecen regularmente en la fase aguda de la enfermedad. Esta se manifiesta por la reducción en el consumo de alimento en postlarva tardía, juveniles y adultos, llegando al punto de un consumo nulo. Posterior a esto, se observan camarones moribundos nadando en la superficie, en la compuerta de salida y en las orillas de los estanques de cultivo; su coloración es de un café amarillento pálido o café oscuro, branquias entre amarillo pálido a café y hepatopáncreas de color café claro a oscuro. En esta fase es cuando se observa la mayor mortalidad, seguida de la fase crónica en la que se observa el hepatopáncreas de color más oscuro y visiblemente atrofiado (Morales \& Cuellar et al. 2014).
El estudio de los OTR en peces comenzó a cobrar importancia a finales de los 1980's, con una incidencia en constante aumento hasta la actualidad. En 1939 se reportó por primera vez un pez de la especie Tetrodon fahaka aparentemente infectado, en el cual se observaron pequeñas formas cocoides dentro de monocitos y plasma (Fryer \& Mauel 1997), aunque no se realizó una confirmación definitiva hasta 1986 cuando se detectó un OTR en cortes histológicos de bazo de Callionymus lyra y se observaron bacterias dentro de células hospederas por medio de microscopía electrónica (Garcés et al. 1991).

Piscirickettsia salmonis es un OTR de importancia en peces de la familia de los salmónidos y es el agente causal de una enfermedad epizoótica en salmónidos, la cual se multiplica dentro de vacuolas citoplasmáticas unidas a la membrana en células huésped (Pulgar et al. 2015). La piscirickettiosis ha sido observada en diversas partes del mundo, entre las que se encuentran Chile, Canadá, Noruega, Irlanda y Escocia. La especie que mayormente ha sido reportada con esta patología es el salmón coho (Oncorhynchus kisutch), aunque también se presenta en el salmón del Atlántico (Salmo salar), trucha

Tabla 2. Especies de crustáceos, infectados con organismos tipo rickettsia (OTR). Obtenida de los reportes de M orado et al. (1988), Nunan et al. (2010), Zewen et al. (2012) y Gollas-Galván et al. (2013) / Crustacean species infected with rickettsialike organisms (RLO). Obtained from the reports of Morado et al. (1988), Nunan et al. (2010), Zewen et al. (2012) and Gollas-Galván et al. (2013)

\begin{tabular}{lll}
\hline $\begin{array}{c}\text { Familias hospederas } \\
\text { de OTR }\end{array}$ & \multicolumn{1}{c}{ Nombre común } & \multicolumn{1}{c}{ Especie } \\
\hline Cancridae & Cangrejo Dungeness & Cancer magister \\
Crangonyctidae & Anfípodo de agua dulce & Crangonyx floridanus \\
Gammaridae & Anfípodo de agua dulce & Rivulogammarus puiex \\
Lithodidae & Cangrejo de Alaska & Lithodes aequispina \\
Lithodidae & Cangrejo rey azul & Paralithodes platypus \\
Nephropidae & Langosta americana & Homarus americanus \\
Pandalidae & Camarón soldado rayado & Plesionika edwardsii \\
Parastacidae & Cangrejo rojo de rio & Cherax quadricarinatus \\
Penaeidae & Camarón Hawaiano & Penaeus marginatus \\
Penaeidae & Langostino banana & Fenneropenaeus merguiensis \\
Penaeidae & Camarón tigre & Penaeus monodon \\
Penaeidae & Camarón blanco del Pacífico & Litopenaeus vannamei \\
Portunidae & Cangrejo verde & Carcinus maenas \\
Portunidae & Cangrejo de la costa Europea & Carcinus mediterraneus \\
Portunidae & Cangrejo azul & Callinectes sapidus \\
Portunidae & Cangrejo azul & Portunus pelagicus \\
Portunidae & Cangrejo azul & Portunus armatus \\
Portunidae & Cangrejo de manglar & Scylla serrata \\
Varinudae & Cangrejo chino & Eriocheir sinensis \\
\hline
\end{tabular}


arcoíris (Oncorhynchus mykiss) y salmón chinook (Oncorhynchus tshawytscha) (Larenas et al. 2005). Los peces afectados presentan branquias pálidas, lo que refleja una anemia severa, corroborada por niveles bajos del hematocrito $(27 \%$, normal $45 \%)$. Externamente se observa oscurecimiento de la piel y abdomen abultado. Uno de los primeros signos a nivel cutáneo es la aparición progresiva de pequeñas induraciones epidérmicas, principalmente en la zona de los flancos y el abdomen (Godoy et al. 2013).

A partir de la publicación de estos nuevos estudios, se ha tenido registro de una gran cantidad de especies de peces infectados por OTR (Tabla 3), lo cual tiene repercusiones no solamente económicas, sino también ambientales, ya que los ecosistemas receptores pueden ser afectados por este tipo de patógenos.

Tabla 3. Especies de peces, infectados con organismos tipo rickettsia (OTR). Obtenida de los reportes de Fryer et al. (1990), Cvitanich et al. (1991), Garcés et al. (1991), Fryer \& Mauel (1997), Almendras et al. (1997) y Gollas-Galván et al. (2013) / Fish species infected with rickettsia like organisms (RLO). Obtained from the reports of Fryer et al. (1990), Cvitanich et al. (1991), Garcés et al. (1991), Fryer \& Mauel (1997), Almendras et al. (1997) and Gollas-Galván et al. (2013)

\begin{tabular}{|c|c|c|}
\hline $\begin{array}{c}\text { Familias hospederas } \\
\text { de OTR }\end{array}$ & Nombre común & Especie \\
\hline Callionymidae & Dragonet & Callionymus lyra \\
\hline Carangidae & Jurel & Trachurus murphyi \\
\hline Cichlidae & Tilapia del Nilo & Oreochromis niloticus \\
\hline Cichlidae & Tilapia barbilla negra & Sarotherodon melanotheron \\
\hline Cichlidae & Jaguar Cichlid & Nandopsis managuense \\
\hline Cyprinidae & Carpa común & Cyprinus carpio \\
\hline Cyprinidae & Piscardo & Pimephales promela \\
\hline Ictaluridae & Siluro marron & Ictalurus nebulosus \\
\hline Loricariidae & Panaque de ojos azules & Panaque suttoni \\
\hline Moronidae & Lubina & Dicentrarchus labrax \\
\hline Salmonidae & Salmón chum & Oncorhynchus keta \\
\hline Salmonidae & Salmón plateado & Oncorhynchus kisutch \\
\hline Salmonidae & Salmón chinook & Oncorhynchus tshawytscha \\
\hline Salmonidae & Salmón chinook & Oncorhynchus tsnewytscne \\
\hline Salmonidae & Salmón rosa & Oncorhynchus gorbuscha \\
\hline Salmonidae & Salmón del Atlántico & Salmo salar \\
\hline Salmonidae & Trucha arco iris & Oncorhynchus myklss \\
\hline Salmonidae & Trucha de lago & Salvelinus namaycush \\
\hline Scianenidae & Lubina blanca & Atractoscion nobilis \\
\hline Scianenidae & Trucha gris & Cynoscion regalis \\
\hline Scophthalmidae & Rodaballo & Scophthalmus aquosus \\
\hline Serranidae & Mero Espaldaron & Epinephelus melanostigma \\
\hline Serranidae & Cabrilla & Paralabrax humeralis \\
\hline Sparidae & Besugo negro & Acanthopagrus schlegeli \\
\hline Sparidae & Besugo rojo & Pagrus major \\
\hline Sparidae & Dorada & Sparus aurata \\
\hline Stromateidae & Palometa estrecha & Peprilus triacanthus \\
\hline Sygnathidae & Frondoso dragón de mar & Phycodurus eques \\
\hline Oplegnathidae & Pez de boca manchada & Oplegnathus punctatus \\
\hline Paralichthyidae & Lenguado común & Paralichthys dentatus \\
\hline Paralichthyidae & Platija Fourspot & Paralichthys oblongus \\
\hline Phycidae & Merluza manchada & Urophycis regia \\
\hline Pleuronectidae & Platija Americana & Hippoglossoides platessoides \\
\hline Pleuronectidae & Lenguado de invierno & Pleuronectes americanus \\
\hline Pleuronectidae & Lenguado cola amarilla & Pleuronectes ferrugineus \\
\hline Tetraodontidae & Pez erizo & Tetrodon fahaka \\
\hline Tetraodontidae & Pez globo de Nilo & Tetraodon lineatus \\
\hline Tetraodontidae & Pez globo japonés & Takifugu rubripres \\
\hline Zoarcidae & Pargo & Macrozoarces americanus \\
\hline
\end{tabular}


El mecanismo de infección de OTR hacia células huésped de organismos acuáticos, no podría llevarse a cabo sin las proteínas de membrana que participan activamente durante todo el proceso de invasión. No obstante, la información con la que se cuenta en relación a este tipo de proteínas de OTR de origen marino es escasa y dispersa. Por lo que el objetivo de esta revisión es sistematizar la información con la que se cuenta en relación a las proteínas transmembranales de OTR de origen marino e inferir sus posibles mecanismos de infección, considerando también la información de otros OTR de origen terrestre que presenten proteínas o genomas similares.

\section{Proteínas de membrana}

Uno de los factores clave del éxito de OTR en la infección de diversos organismos acuáticos son sus proteínas de membrana, las cuales juegan un rol medular en el proceso de infección. Las proteínas de membrana poseen una orientación definida en relación con el citoplasma, estas pueden tener una proyección interna (citosol) o externa y llevar a cabo distintas funciones (Karp 2013). Este tipo de proteínas pueden agruparse de acuerdo a su relación con la bicapa lipídica; por ejemplo, a las proteínas que penetran la bicapa lipídica se les conoce como transmembranales o integrales, mientras que las proteínas que se encuentran en su totalidad fuera de la bicapa lipídica, reciben el nombre de proteínas periféricas. Por último, se encuentran las proteínas fijadas a lípidos, o también llamadas lipoproteínas situadas dentro de la célula y unidas a la bicapa lipídica mediante enlaces covalentes (Karp 2013, Brocks et al. 2014).

Específicamente las proteínas transmembranales o integrales, atraviesan ambos extremos de la membrana celular, con dominios que sobresalen por ambos lados de la misma. Éstas cumplen distintas funciones: como receptores, como canales o transportadores implicados en el movimiento de iones y solutos a través de la membrana y como receptores que reconocen y fijan determinadas moléculas llamadas ligandos, para la identificación de una hormona, neurotransmisor o nutriente (Karp 2013).

Las proteínas transmembranales de organismos tipo rickettsia (OTR), además de participar en el transporte como receptoras de fagos, también desempeñan un papel importante en la patogenicidad. Estas proteínas han sido utilizadas para la clasificación de organismos a nivel de género; un ejemplo de lo anterior es el análisis de antígenos de superficie celular 0 y 5 (Sca0 y Sca5) también conocidos como OmpA y OmpB respectivamente, los cuales permiten diferenciar al género rickettsiae en dos grupos: el grupo de la fiebre manchada y el grupo tifo.

El grupo de la fiebre manchada contiene exclusivamente Sca0 que transcribe una proteína de membrana externa de 170-198 $\mathrm{kDa}$, la cual es suficiente para mediar en la adhesión y la invasión de células huésped (Riley et al. 2010, Chan et al. 2011), esto es logrado por la estimulación de la polimerización de actina como medio de propulsión e invasión de células adyacentes, en la presencia de un lipopolisacárido (LPS) específico (Valbuena 2010).

El grupo tifo, además de contener Sca0, también está integrado por Sca5 (Andrade 2010) que transcribe una proteína de membrana externa de $120-168 \mathrm{kDa}$, la cual funciona como ligando del receptor bacteriano a Ku70 de la célula huésped, por lo tanto, juega un papel importante en el establecimiento de una infección exitosa dentro de las células diana; un ejemplo de ello se ha observado en mamíferos, donde además de producirse la interacción Sca5-Ku70, también se puede dar el caso de interacciones de Sca5 con los receptores de la célula huésped Ku80, Ku86 y PARP para formar el complejo ADNPK (Martínez 2015).

Las proteínas de membrana externa se consideran como antígenos inmuno expuestos que sufren constantes mutaciones, lo cual podría ser una herramienta de diferenciación entre las especies de los OTR, logrando así una mejor clasificación (Gong et al. 2014).

Se ha demostrado que Sca0 (OmpA) y Sca5 (OmpB) son importantes para la adhesión y la invasión posterior de células de mamífero no fagocíticas y en la generación de respuestas inmunes humorales de protección (junto con Sca1 y Sca2); incluso se ha identificado la primera proteína-receptor de la célula huésped para OTR (Sca5-Ku70). A pesar de que parte de esta información se extrae de estudios en mamíferos, proteínas similares se han identificado en OTR reconocidos como patógenos para organismos acuáticos como peces y crustáceos (Chan et al. 2009), con lo cual se asume que el mecanismo de adhesión es similar.

\section{MeCANISMO DE INFECCIÓN}

El mecanismo de infección de OTR hacia la célula huésped es usualmente conservado entre huéspedes pertenecientes a distintos grupos taxonómicos (Uchiyama 2012). El proceso de invasión inicia con la adhesión entre el receptor Ku70 de la célula huésped con el receptor bacteriano llamado antígeno de superficie celular 5 (Sca5), también conocido como OmpB (Chan et al. 2009); posteriormente ocurre la adhesión de antígenos de superficie celular como $\mathrm{Sca} 0$ conocido también como OmpA, que participa en la adhesión e invasión celular y Sca1 que participa solamente en la adherencia de la célula huésped (Riley et al. 2010). Una vez que ha ocurrido la adhesión, el sistema de secreción tipo 4 (T4SS, por sus siglas 
en inglés Type IV Secretion Systems) inyecta efectores como Sec7, LepA, LepB y patatinas en la célula diana para apoderarse de diversos mecanismos celulares (La et al. 2007). Algunas enzimas llamadas translocasas participan en el intercambio de diversos componentes entre la bacteria y la célula huésped; por ejemplo la translocasa 1 (Tlc1) intercambia ADP (Adenosindifosfato) bacteriano por ATP (Adenosintrifosfato) de la célula huésped con el fin de obtener energía, la translocasa 4 (Tlc4) interviene en el transporte de CTP (Citidinatrifosfato) y UTP (Uridinatrifosfato) hacia la bacteria y la translocasa 5 (Tlc5) se encarga de transportar GTP (Guanosintrifosfato) hacia la bacteria (Audia \& Winkler 2006).

Posterior a la adhesión ocurre la incorporación de la bacteria hacia la célula huésped por medio de un proceso de fagocitosis; la célula huésped adopta este comportamiento por la interacción de algunos efectores que modifican la función de las proteínas, como ubiquitina, Caveolina 2 y Clatrina, todas responsables de regular diversos procesos celulares, provocando un rearreglo de la membrana celular y la consiguiente invasión bacteriana (Martínez et al. 2005). Al encontrarse la bacteria en el endosoma (también llamado fagosoma) dentro del citosol de la célula huésped, la bacteria escapa del fagosoma lisándolo con la ayuda de las proteínas fosfolipasa D (Pld) y fosfolipasa C (TlyC) (Whitworth et al. 2005). La bacteria ya libre en el citosol, utiliza la proteína RicA y Sca2 para reclutar a la proteína Arp2/3 y polimerizar actina, con el fin de obtener motilidad dentro del citosol, logrando con ello poder trasladarse e invadir el núcleo celular o alguna célula vecina, donde podrá llevar a cabo su replicación por fisión binaria (tanto en la célula vecina, el citosol o el núcleo celular), para después lisar la membrana de la célula huésped gracias a la citotoxicidad producida por la bacteria con la ayuda de algunos efectores (Sec7, LepA, LepB y patatinas). La forma en que ocurre la lisis celular no se encuentra completamente dilucidada, por lo que es necesario realizar un mayor número de investigaciones (Uchiyama 2012).

\section{Proteínas transmembranales}

Las proteínas transmembranales son un tipo de proteínas de membrana que abarcan la totalidad de la membrana biológica a la que están unidos de forma permanente, es decir, las proteínas transmembrana atraviesan de un lado al otro la membrana. Son proteínas anfipáticas y están divididas en tres partes. La primera sección atraviesa la membrana, por lo que tiene un carácter hidrofóbico; es por ello que su interacción es con los lípidos de la bicapa lipídica que se encuentra formando parte de la membrana celular. La segunda sección se encuentra en contacto con el interior celular, por lo cual se le conoce como dominio citosólico; esta sección es de carácter hidrofílico. La tercera parte se encuentra en contacto con el exterior de la célula, es conocida como dominio extra citosólico, de carácter hidrofílico al igual que la sección anterior. También, se conoce que las proteínas transmembrana son proteínas politópicas que tienden a agregarse y precipitar en agua. Requieren detergentes o disolventes no polares para la extracción, aunque algunas de ellas (ß-barril) pueden ser también extraídas por medio de agentes desnaturalizantes (Lodish et al. 2005, Goodman 2010).

Existen dos tipos de proteínas transmembranales según su función: las proteínas unipaso y las multipaso. Las proteínas unipaso como su nombre lo indica, son aquellas que atraviesan la bicapa lipídica una sola vez, es decir, el extremo N-terminal queda de un lado de la membrana y el extremo C-terminal del otro lado. Las proteínas transmembranales multipaso, atraviesan la bicapa lipídica en dos o más ocasiones, normalmente mediante varias $\beta$-hélices, aunque hay casos de inserciones a través de ß-lámina (Karp 2013).

Dentro de las proteínas transmembranales multipaso, se encuentran las proteínas de autotransporte, las cuales tienen estructuras modulares, incluyendo un péptido de señal $\mathrm{N}$ terminal (SP), un dominio pasajero central y un módulo de translocación C-terminal llamado ß-péptido como se observa en la Figura 1. Después de la traducción, el polipéptido es secretado a través de la membrana interna por el translocón Sec dentro del espacio periplásmico, usando información codificada en la secuencia de señal N-terminal. A través de un mecanismo definido, los $\beta$-péptidos se insertan en la membrana externa bacteriana, para formar un poro transmembrana $\beta$-barril para la secreción del dominio central (pasajero) en el espacio extracelular. En el caso de algunas proteínas, por ejemplo OmpB, el péptido señal posteriormente es proteolizado de la porción de $\beta$-peptido de la proteína y de esta manera permanece asociado en el exterior de la membrana externa de los OTR (Chan et al. 2011).

\section{Proteínas transmembranales Que participan en el PROCESO DE INFECCIÓN}

Las infecciones por OTR son causadas por una variedad de bacterias de los géneros Rickettsia, Orientia, Ehrlichia, Neorickettsia, Neoehrlichia, y Anaplasma. Los OTR de importancia en la patogenicidad de organismos acuáticos se dividen en dos grupos; los grupos TG y SFG, siendo principalmente reportadas en especies de bivalvos, peces y crustáceos, muchas de ellas de suma importancia en la industria acuícola (McQuiston 2016).

Algunos OTR que infectan a peces poseen Sca0 también conocido como OmpA, mientras que otros presentan Sca5 reportado también como OmpB y algunos otros ambas (Wu 2003, McQuiston 2016). Una de las bacterias intracelulares 


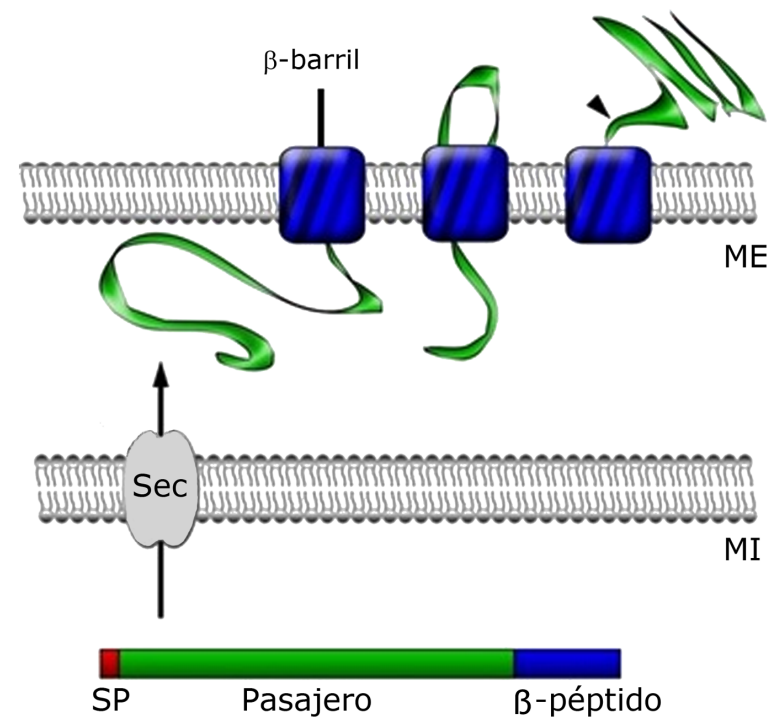

Figura 1. Estructura y secreción de proteínas autotransportadoras. Modificado de Chan et al. (2010). ME: Membrana externa; M I: Membrana interna; SP: Señal peptídica N-terminal; Sec: Translocón / Structure and secretion of autotransporter proteins. Modified from Chan et al. (2010). ME: Outer membrane; MI: Internal membrane; SP: Signal peptide N-terminal; Sec: Translocon

más documentadas en organismos acuáticos es Piscirickettsia salmonis, un microorganismo altamente virulento que afecta a la salmonicultura (Fryer \& Hedrick 2003, Pulgar et al. 2015). Se han realizado comparaciones en base a la secuencia de aminoácidos KGP96444.1 correspondiente al Sca0 de $P$. salmonis y a la secuencia AAG43542.1 de Sca5 de Rickettsia prowazekii, con secuencias registradas en el GenBank, de lo cual se obtiene que algunos OTR poseen ambos tipos de Sca, además de que aparentemente Sca5 tiende a ser una región mucho más conservada; aunque esto último solo representa una hipótesis que debe ser probada con más estudios de este tipo (Tabla 4). Sin embargo, existe evidencia bioinformática recabada en nuestro laboratorio, que sugiere que los mecanismos de invasión podrían ser altamente conservados entre OTR terrestres y acuáticos (datos no publicados).

Se ha identificado una familia de al menos 17 genes llamados Sca; el producto de estos genes son proteínas de adherencia y de autotransporte, las cuales participan en el proceso de invasión e infección. Estas proteínas también participan en la motilidad que le confiere la polimerización de actina dentro de la célula huésped y están integradas por un péptido de señal N-terminal, un dominio de pasajero y un módulo de translocación $\mathrm{C}$ terminal (ß-péptido). Después de la traducción, el dominio Cterminal se inserta en la membrana externa formando un poro, permitiendo la exposición externa del dominio de pasajero. En la mayoría de los OTR del grupo SFG se han reportado cinco genes muy conservados de la familia Sca (ScaO u OmpA, Sca1, Sca2, Sca4 y Sca5 u OmpB), lo cual representa evidencia de su importante rol en el ciclo de vida de la bacteria (Blanc et al. 2005, Chan et al. 2011, Riley et al. 2010, Kleba et al. 2010).

\section{SCA0 (ОмРA)}

El gen que codifica para Sca0 (OmpA) se encuentra conservado en todo el grupo de SFG y está ausente en OTR del grupo TG (Blanc et al. 2005). Las SFG se caracterizan por tener antígenos de superficie celular 0 ( $\mathrm{Sca} 0$ ) también conocidas como proteínas de membrana externa tipo A (OmpA) de aproximadamente $135-247 \mathrm{kDa}$, las cuales presentan un punto isoeléctrico $(\mathrm{pI})$ de 5.1 y contienen un dominio de 6 a 15 unidades similares repetidas en tándem. Este tipo de proteínas se han reportado en más de 23 especies de rickettsias (Noriea et al. 2015). Por ejemplo, se conoce que en $R$. rickettsii cepa Sheila Smith, Sca0 codifica para una proteína de $247 \mathrm{kDa}$, mientras que en $R$. conorii Malish 7, Sca0 codifica para una proteína de membrana de $224 \mathrm{kDa}$ y en $R$. rickettsii cepa lowa, Sca0 codifica una proteína de $247.390 \mathrm{kDa}$, es decir, las longitudes de los antígenos superficiales Sca0 varían de una especie a otra y con ello también varían sus pesos moleculares, desde 135-247 kDa aproximadamente, estas diferencias se deben en gran parte a las variaciones en el número de secuencias 
Tabla 4. Comparación en base a las proteínas OmpA de Piscirickettsia salmonis con número de acceso KGP96444.1 y a la proteína OmpB de Rickettsia prowazekii con número de acceso AAG43542.1, con las proteínas OmpA y OmpB detectadas respectivamente en diferentes organismos tipo ricketssia / Comparison based on proteins OmpA Piscirickettsia salmonis accession number KGP96444.1 and to protein OmpB Rickettsia prowazekii accession number AAG43542.1, with OmpA and OmpB detected respectively in different rickettsia-like organisms

\begin{tabular}{lccccc}
\hline \multicolumn{1}{c}{ Bacteria } & Número de acceso & a.a & pI & $\begin{array}{c}\text { Tipo de } \\
\text { Omp }\end{array}$ & $\begin{array}{c}\% \text { de } \\
\text { Similitud }\end{array}$ \\
\hline Piscirickettsia salmonis & KGP96444.1 & 214 & 6.424 & OmpA & $100 \%$ \\
\hline Diplorickettsia massiliensis & WP_010597033.1 & 189 & 7.629 & OmpA & $25 \%$ \\
Sutterella parvirubra & WP_008543693.1 & 268 & 7.543 & OmpA & $27 \%$ \\
Coxiella burnetii & NP_819350.1 & 231 & 1.196 & OmpA & $27 \%$ \\
Rickettsia rickettsia & ADO24533.1 & 1045 & 5.047 & OmpA & $40 \%$ \\
Rickettsia conorii & AAA99908.1 & 1029 & 5.193 & OmpA & $23 \%$ \\
Rickettsia slovaca & AFK77692.1 & 152 & 4.102 & OmpA & $36 \%$ \\
Rickettsia australis & AAD39531.2 & 2106 & 4.735 & OmpA & $26 \%$ \\
\hline Rickettsia prowazekii & AAG43542.1 & 1643 & 5.064 & OmpB & $100 \%$ \\
\hline Rickettsia typhi str. Wilmington & AAU04158.1 & 1645 & 5.199 & OmpB & $87 \%$ \\
Rickettsia rickettsii & CAA34403.1 & 1654 & 5.780 & OmpB & $71 \%$ \\
Rickettsia conorii & AAD39533.1 & 1303 & 5.861 & OmpB & $72 \%$ \\
Rickettsia slovaca & AFW90548.1 & 766 & 5.126 & OmpB & $70 \%$ \\
Rickettsia australis & AAD39532.1 & 1304 & 5.524 & OmpB & $71 \%$ \\
\hline
\end{tabular}

OmpA: Proteína de membrana externa A (Outer membrane protein A)

OmpB: Proteína de membrana externa B (Outer membrane protein B)

a.a: Aminoácidos

pI: Punto isoeléctrico

repetidas en tándem presentes en la región de dominio de pasajero de la proteína, lo cual sugiere una posible adaptación a condiciones diversas (Ellison et al. 2008, Chan et al. 2011, Clark et al. 2015).

Estudios han sugerido que Sca0 juega un papel en la adhesión al huésped, pero no es crítica para la virulencia (Chan et al. 2009, Noriea et al. 2015). Aunque poco se conoce sobre la función de estas regiones repetidas en tándem, su presencia y conservación dentro de algunos OTR del grupo SFG sugiere una función conservada en esta proteína, como la adhesión a células huésped (Chan et al. 2011); sin embargo aún se requieren estudios enfocados a elucidar dicha información.

\section{Sca1}

El antígeno de superficie celular 1 (Sca1) es una proteína de membrana externa de la mayoría de los OTR del grupo SFG, con características de adherencia y autotransporte al igual que Sca0 y Sca5. El tamaño total de Sca1 varía ampliamente entre rickettsias, desde 594 hasta 1976 aminoácidos, es decir, de 65-217 kDa y un pI de 10.22 (Riley et al. 2010); sin embargo, ha conservado sus dominios específicos a pesar de verse limitado por la presión selectiva en la diversidad de sus regiones N-terminal y ß-péptidos (Ngwamidiba et al. 2005).

Se ha demostrado en cultivo de células de mamíferos que Sca1 se expresa en la superficie de $R$. conorii, con un peso molecular aproximado de $120 \mathrm{kDa}$, muy parecido al peso molecular de Sca5. También existe evidencia de que Sca1 tiene un papel en la adherencia a las células huésped, aunque no a la invasión, ya que son eventos distintos que pueden ser mediados por diversas proteínas de membrana externa de rickettsias SFG (Riley et al. 2010) y aunque no existen reportes relacionados con la caracterización de Sca1 en OTR que afectan organismos acuáticos, al ser este tipo de patógenos generalmente rickettsias SFG, podría esperarse que antígenos como Sca1 y otros antígenos del tipo Sca, estén presentes en OTR de ambientes acuáticos (Küchler et al. 2009). A este respecto, un estudio reciente, reveló que el transcriptoma de un OTR aislado del alga verde Mesostigma viride presentaba transcritos que codifican para proteínas transmembranales tipo Sca, las cuales juegan un rol en la adherencia (Yang et al. 2016). 


\section{ScA 2}

El antígeno de superficie celular 2 (Sca2) es una proteína autotransportadora y de adherencia, se encuentra presente en OTR del grupo SFG. El gen codifica para una proteína de aproximadamente $200-220 \mathrm{kDa}$, con un pI de 8.66, tiene una similitud en sus aminoácidos del 80 a $95 \%$ entre las diferentes especies de OTR del grupo SFG. Esta proteína participa en la infección y ayuda a la motilidad de actina en la célula huésped (Kleba et al. 2010). Este antígeno de superficie funciona junto con otras moléculas como adhesinas de diversos receptores que se han encontrado en células endoteliales de los vasos sanguíneos en mamíferos. Este proceso induce a la fagocitosis la cual no es una función común en este tipo de células; esto se logra induciendo cambios en el citoesqueleto de actina provocando la inclusión bacteriana. Este mecanismo solo se ha observado en células de mamíferos, pero se considera que podría ser similar para las células de diversos tejidos de animales acuáticos infectados con OTR. Lo anterior se desprende del hecho de que los OTR tienen genes estrechamente conservados y particularmente en este tipo de proteínas de membrana, por lo que se espera una similitud en cuanto al proceso de infección de OTR de organismos acuáticos y mamíferos (Martínez \& Cossart 2004). Es necesario realizar más estudios para corroborar la hipótesis anterior, una primera aproximación podría darse al utilizar la información de los genomas registrados en las bases de datos de este tipo de bacterias; buscando la presencia de genes que codifiquen para antígenos de superficie y su similitud con otros genes de OTR de mamíferos.

\section{Sca4}

El antígeno de superficie celular 4 (Sca4) de OTR es una proteína autotransportadora, con un tamaño que oscila entre los 142-273 kDa y un pI de 4.86. Esta proteína participa en la invasión de la célula huésped relacionándose con vinculina e interactuando con los sitios de unión a vinculina (VBS) que se conservan en todos los OTR (Chan et al. 2009). Sorprendentemente, esto ocurre a través de mimetismo molecular de la interacción vinculina-talina. Las estructuras cristalinas vinculina-VBS de Sca4 revelan que la vinculina adopta una nueva conformación cuando se une a los VBS C-terminales de Sca4 (Park et al. 2011). Aunque este tipo de proteína no se ha identificado todavía en OTR de ambientes acuáticos o marinos, estudios previo basados en análisis filogenéticos han demostrado que la mayor parte de las bacterias tipo rickettsia cuentan con Sca4 o proteínas similares (Guillespie et al. 2008).

\section{Sca5 (OMpB)}

A este tipo de proteínas anteriormente se les conocía como SpaP, SPA o p120, las cuales tienen un peso molecular aproximado de 120-168 kDa y un pI de 4.64; éstas son proteínas de membrana externa con diferentes funciones como adherencia, invasión y autotransporte. Contienen múltiples dominios transmembranales y se encuentran formando parte de los dos grupos de OTR de importancia para organismos acuáticos, es decir, TG y SFG (Chan et al. 2009). Este tipo de proteínas de membrana se consideran comoel tipo de antígeno inmunodominante de la mayoría de los OTR (Roux \& Raoult 2002). Sca5 se expresa como una pre-proteína de 168 kDa y se divide liberando el dominio de pasajero de $120 \mathrm{kDa}$ desde el dominio de translocación de $\beta$-barril de $32 \mathrm{kDa}$, que después expone el dominio maduro de $120 \mathrm{kDa}$ a la parte exterior de la membrana externa (Fig. 1) (Chan et al. 2011). En estudios realizados por Blanc y colaboradores (2005), se observó un alto nivel de conservación del Sca5 entre los diversos grupos de OTR, mostrándose una similitud del 70 al $95 \%$ en sus secuencias de aminoácidos. También se ha observado a través de un método cromatográfico de afinidad, que Sca5 es un ligando del receptor bacteriano Ku70 de la célula huésped, lo que sugiere que éstas funcionan como un par de adhesinareceptor en la interacción celular OTR-hospedero. Lo anterior podría considerarse como evidencia sólida de que el área conservada de Sca5 cumple una función en la patogenicidad de la bacteria (Martínez et al. 2005, Chan et al. 2009).

Evidencia reciente demostró que un OTR aislado de algas verdes (Mesostigma viride) presenta genes que codifican para Sca5, así como para los receptores Ku70, WASH, SCAR y CDC42 (Yang et al. 2016). Además, se encontró evidencia de sub unidades de Ku70 en una amplia diversidad de organismos marinos tanto vertebrados como invertebrados (Kanungo et al. 1999, Pereira et al. 2011, Rhee et al. 2012).

\section{Translocasas}

Otras de las proteínas de membrana externa encontradas en OTR son las enzimas llamadas translocasas, también se han identificado en las mitocondrias y plástidos. Los OTR se han considerado patógenos obligados debido al transporte de muchos compuestos esenciales (es decir, aquellos que no pueden sintetizar) desde el citoplasma de la célula huésped. Se ha reportado actualmente que en $R$. prowazekii, al igual que otros OTR, codifican cinco diferentes translocasas, algunas con funciones ya definidas y otras aún por definir. Una de las translocasas más estudiadas es la translocasa 1 (Tlc1) cuya función es intercambiar ADP bacteriano por ATP de la célula huésped como fuente de energía para la bacteria, posee un P.M. de 9-99 kDa y un pI de 9.03 (Audia \& Winkler 2006). Es una proteína transportadora que permite que el ATP y ADP atraviesen las membranas de los OTR. Más del 10\% de la proteína en la membrana mitocondrial interna consta de translocasa ATP-ADP (Stryer et al. 2007). 
Por otro lado, aunque se ha detectado la expresión de translocasa 2 (Tlc2) y translocasa 3 (Tlc3) su función aún no está bien definida (Kleba et al. 2010). En el caso de la translocasa 4 (Tlc4) es una proteína autotransportadora, con un P.M. entre 8-100kDa y un pI cercano a 9.92, se cuenta con cierta evidencia que indica que interviene en el trasporte de CTP y UTP hacia los OTR. La translocasa 5 por su parte (Tlc5) al igual que las translocasas anteriores, es una proteína autotransportadora con un tamaño de 9-97 kDa y un pI de 9.583. Se encarga de transportar GTP hacia los OTR y GDP a la célula huésped, con lo cual se puede concluir que Tlc4 y Tlc5 cumplen con la función primordial de mantener la entrada de nucleótidos para la biosíntesis de ácidos nucleicos, como la Tlc1 lo realiza con el ATP-ADP usándolo como fuente de energía (Audia \& Winkler 2006).

Se ha observado en camarones cultivados un agente infeccioso bacteriano muy similar a OTR que afecta a algunos crustáceos como el camarón blanco Litopenaeus vannamei, llamada Bacteria de la Necrosis Hepatopancreática (BNHP) o Candidatus Hepatobacter penaei (Nunan et al. 2013), la cual tiene una similitud de alrededor del $83.5 \%$ en sus nucleótidos con el grupo de los OTR, es decir, existe una notable cercanía filogenética, tanto que podrían catalogarse dentro del mismo grupo. Considerando lo anterior, es probable que en ellas se encuentren ciertas proteínas de membrana externa tipo Sca conocidas también como Omp (Sca0 o OmpA, Sca1, Sca2, Sca5 o OmpB) y Translocasas (Tlc1, Tlc2, Tlc3, Tlc4 y Tlc5). En la Tabla 5, se muestra una comparación de la secuencia
U65509 (número de acceso para BNHP) con todas las secuencias presentes en el GenBank. Se modificaron los datos de la tabla realizada por Gollas-Galván et al. (2013) con el fin de actualizar y delimitar los datos, empleando las herramientas del NCBI, es decir, se delimita la comparación en similitud solo por encima del $85 \%$ y se realizó la actualización de números de accesos y especie de algunos organismos.Gracias a la gran cantidad de trabajos realizados en vertebrados sobre los diferentes tipos de proteínas de membrana externa, se ha llegado a entender cómo es que se logra la adherencia e invasión de los OTR a las células huésped (Fig. 2). Se conoce que la interacción de estas múltiples adhesiones contribuye a la activación de una serie de vías de señalización, que reclutan actina para la penetración y movimiento del patógeno por la célula huésped. Aunque este modelo de invasión no representa la totalidad del proceso de infección, si provee de una base para entender el mecanismo de infección y de esta manera poder actuar en consecuencia inhibiendo la interacción huésped-patógeno, lo que evitaría la enfermedad.

Aunque la mayoría de los estudios se han realizado sobre enfermedades en vertebrados y no sobre invertebrados acuáticos; se presupone que el modo de infección sea equivalente, ya que los genomas de distintas especies de rickettsias son altamente conservados, por lo que se espera que codifiquen para proteínas similares, con participación semejante en los mecanismos de infección (Wang \& Gu 2002). La presencia de algunas translocasas tales como ADP/ATP translocasa parece tener una distribución universal en patógenos

Tabla 5. Similitud de BNHP basada en la comparación de la secuencia U65509* / Similarity of NHPB based on comparison with the sequence U65509*

\begin{tabular}{|c|c|c|c|}
\hline $\begin{array}{l}\text { Porcentaje de } \\
\text { similitud }\end{array}$ & Número de acceso & Organismo & Fuente \\
\hline 88 & CU926702.1 & $\alpha$-proteobacteria no cultivada & Muestras de ambientes lodosos \\
\hline 88 & EF575101.1 & Clon de bacteria no cultivada & $\begin{array}{l}\text { Microorganismos marinos de la Isla } \\
\text { del Coco }\end{array}$ \\
\hline 87 & AB294318.1 & Gen de bacteria no cultivada & $\begin{array}{l}\text { Aguas subterráneas profundas del } \\
\text { norte de Japón }\end{array}$ \\
\hline 89 & NR_044847.3 & Caedibacter caryophilus & Paramecium caudatum \\
\hline 87 & AM $\overline{2} 36093$ & Caedibacter macronucleorum & Paramecium duboscqui \\
\hline 87 & FJ205252.1 & $\begin{array}{l}\text { Clon } 1 \text { de } \alpha \text {-proteobacteria no } \\
\text { cultivada }\end{array}$ & Región hidrotérmica de alta mar \\
\hline 88 & DQ501339.1 & $\alpha$-proteobacteria no cultivada & Lagos del noreste de Alemania \\
\hline 85 & NR_116679.1 & Oceanibaculum pacificum & $\begin{array}{l}\text { Sedimentos campo hidrotermal del } \\
\text { suroeste del Océano Pacífico }\end{array}$ \\
\hline
\end{tabular}

* Reproducida con permiso de Gollas-Galván et al. (2013), con actualizaciones de números de accesos y especie de algunos organismos. Solo se consideran similitudes por encima del $85 \%$ en base a secuencia de aminoácidos 


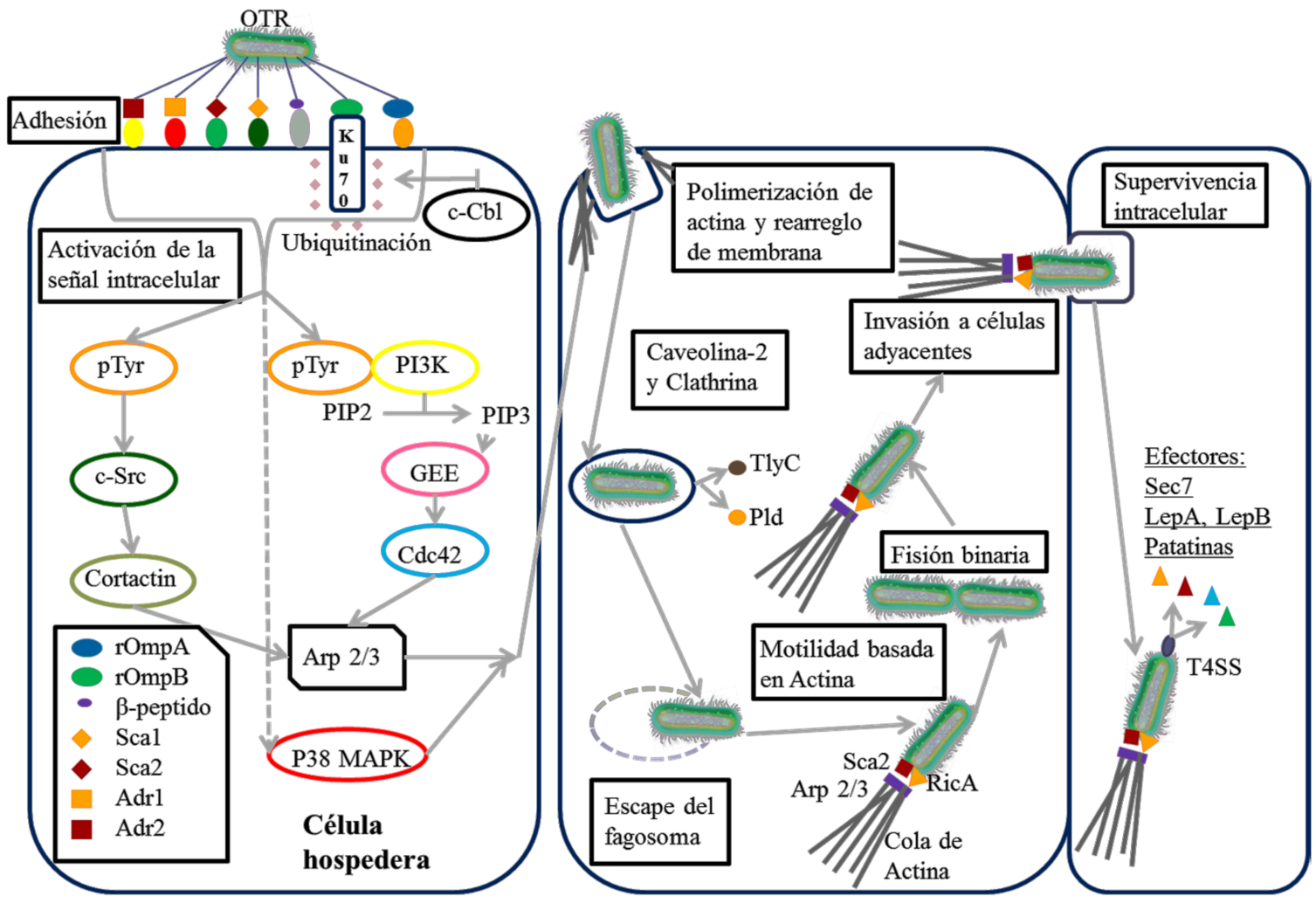

Figura 2. Modelo de invasión de algunos OTR a célula huésped no fagocítica (Modificada de Wang \& Gu 2002, Uchiyama 2012) / Invasion model of RLO to non-phagocytic host cells (Modified from Wang \& Gu 2002, Uchiyama 2012)

intracelulares como OTR y organismos tipo clamidia (OTC), incluyendo aquellos de origen marino (Greub \& Raoult 2003, Schmitz-Esser et al. 2004).

Sin embargo, la información en relación a las proteínas transmembranales de OTR en organismos marinos es escasa, por lo que es importante realizar investigaciones para fortalecer las bases de datos que permitan tener un mayor conocimiento sobre la diversidad de bacterias patógenas, en organismos acuáticos de importancia comercial y de esta manera poder actuar contra los problemas existentes.

Aunque la información de proteínas transmembranales de OTR es escasa, esta pudo ser complementada con la información de OTR de origen terrestre, ya que comparten muchas proteínas conservadas, lo cual da cabida para asumir de manera hipotética que el mecanismo de infección de OTR de origen terrestre es similar al de OTR de origen marino.

Uno de los retos a futuro en el estudio de OTR de organismos acuáticos o marinos, es el diseño de medios de cultivo celular, ya que la mayor parte de este tipo de bacterias intracelulares no es cultivable. Se han logrado algunos cultivos utilizando líneas celulares, sin embargo esto no garantiza el total aislamiento del patógeno (Luce et al. 2015). Así mismo, se espera que el surgimiento de las tecnologías de secuenciación de siguiente generación y nuevas tecnologías para el estudio de la proteómica, representen un mayor avance en el conocimiento de OTR de origen marino.

\section{Conclusiones}

Considerando la evidencia recabada, el mecanismo de adherencia, invasión e infección de OTR a las células huésped es un modelo que bien puede ocurrir en OTR de origen marino. Las similitudes detectadas en los componentes que juegan un rol en el proceso de infección, además de la alta conservación del genoma de los OTR, hace suponer que OTR de origen marino codifiquen para proteínas similares, con participación semejante en los mecanismos de infección. 
La información recabada sobre OTR de organismos acuáticos o terrestres, permite inferir el mecanismo de patogenicidad en el que intervienen cada una de las proteínas transmembranales, desde la adhesión inicial mediada por Sca5 de OTR con Ku70 de la célula huésped, seguida por la activación de Sca0, Sca1, Sca2 y Sca4 que interactúan con receptores de adhesión y receptores de invasión de la célula huésped provocando la fagocitosis. Esto ocurre a pesar de que la célula huésped no sea una célula fagocítica; sin embargo, esto da cabida a la bacteria dentro del citosol en un fagosoma o endosoma, que es posteriormente lisado por hemolisina $\mathrm{C} \mathrm{y}$ fosfolipasa D. Una vez ocurrido esto, la bacteria esta lista para su replicación por fisión binaria ya sea en el mismo citosol o puede emigrar al núcleo de la célula afectada ya que RickA y ARP 2/3 le confieren movilidad gracias a la polimerización la actina. El mecanismo anteriormente descrito es posible gracias a la intervención de la familia de las translocasas (Tlc) las cuales ayudan a obtener diversos tipos de nucleótidos como ATP, CTP, UTP y GTP; ya que los OTR no los pueden sintetizar, convirtiendo a los OTR en parásitos intracelulares obligados, ayudados por el sistema de secreción tipo 4 (T4SS), para inyectar proteínas efectoras y apoderarse de la maquinaria de la célula huésped para sus propios fines. Con lo anteriormente descrito se espera tener una mejor visión sobre el mecanismo de infección de los OTR con el fin hacerles frente a las enfermedades de una forma directa y certera, evitando así su propagación, efectos secundarios y resistencia del patógeno.

\section{Agradecimientos}

Al Consejo Nacional de Ciencia y Tecnología (CONACyT), por el apoyo económico tanto para la beca estudiantil, como para los insumos y consumibles (Proyecto Ciencia Básica: 168614). Al Departamento de Investigación Científica y Tecnológica de la Universidad de Sonora, por dejarme ser parte de sus programas de posgrado. Al Centro de Investigación en Alimentos y Desarrollo (CIAD) A.C., especialmente a la Coordinación de Tecnología de Alimentos de Origen Animal por las facilidades que se me brindaron (Jesús Pérez-Acosta).

\section{LITERATURA CITADA}

Almendras F, S Jones, C Fuentealba \& G Wright. 1997. In vitro infection of a cell line from Ictalurus nebulosus with Piscirickettsia salmonis. Canadian Journal of Veterinary Research 61: 66-68.

Andrade 0. 2010. Amblyomma imitator: a novel vector for Rickettsia rickettssi in México and south Texas. Tesis Doctor Scientae, Universidade Federal de Viçosa, Viçosa, $28 \mathrm{pp}$.
Audia P \& H Winkler. 2006. Study of the five Rickettsia prowazekii proteins annotated as ATP/ADP Translocases (Tlc): only Tlc1 transports ATP/ADP, while Tlc4 and Tlc5 transport other ribonucleotides. Journal of Bacteriology 188: 6261-6268.

Azevedo C, R Conchas, J Tajdari \& J Montes. 2006. Ultrastructural description of new Rickettsia-like organisms in the commercial abalone Haliotis tuberculata from the NW of Spain. Diseases of Aquatic Organisms 71: 233-237.

Azevedo C, I Mendonca \& E Matos. 2005. Ultrastructural analysis of rickettsia-like organisms in the oyster Crassostrea rizophorae from the northeastern Atlantic coast of Brazil. Laboratory, Federal Rural University of Amazonia (UFRA), Belém, PA. Brazil. Brazilian Journal of Morphological Sciences 22: 5-8.

Blanc G, M Ngwamidida, H Ogata, P Fournier, J Claverie \& D Raoult. 2005. Molecular evolution of rickettsia surface antigens: Evidence of positive selection. Molecular Biology and Evolution 22: 2073-2083.

Brooks G, K Carroll, J Butel, S Morse \& T Mietzner. 2014. Microbiología médica, 833 pp. McGraw-Hill, Houston.

Ceuta L \& G Boehs. 2012. Parasites of the mangrove mussel Mytella guyanensis (Bivalvia: Mytilidae) in Camamu Bay, Bahia, Brazil. Brazilian Journal of Biology 72: 421-427.

Chan YG, MM Cardwell, TM Hermanas, T Uchiyama \& JJ Martinez. 2009. Rickettsial outer-membrane protein B $(\mathrm{rOmpB})$ mediates bacterial invasion through $\mathrm{Ku} 70$ in an actin, c-Cbl, clathrin and caveolin 2-dependent manner. Cell Microbiology 11: 629-644.

Chan YGY, SP Riley \& JJ Martínez. 2010. Adherence to and invasion of host cells by spotted fever group rickettsia species. Frontiers in Microbiology 1(139), <doi:10.3389/ fmicb.2010.00139>

Chan YGY, SP Riley, E Chen \& JJ Martínez. 2011. Molecular basis of immunity to rickettsial infection conferred through outer membrane protein B. Infection and Immunity 79(6): 2303-2313.

Clark T, N Noriea, D Bublitz, D Ellison, C Martens, E Lutter \& T Hackstadt. 2015. Comparative genome sequencing of Rickettsia rickettsii strains that differ in virulence. Journal of Infection and Immunity 83: 1568-1576.

Cvitanich J, O Garate \& C Smith. 1991. The isolation of a rickettsia-like organism causing disease and mortality in Chilean salmonids and its confirmation by Koch's postulate. Journal of Fish Diseases 14: 121-145.

Ellison D, T Clark, D Sturdevant, K Virtaneva, S Porcella \& T Hackstadt. 2008. Genomic comparison of virulent Rickettsia rickettsii Sheila Smith and avirulent Rickettsia rickettsii Iowa. Infection and Immunity 76: 542-550.

Ferrantini F, S Fokin, L Modeo, I Andreoli, F Dini \& H Gortz. 2009. 'Candidatus cryptoprodotis polytropus' A novel Rickettsia-like organism in the ciliated protist Pseudomicrothorax dubius (Ciliophora, Nassophorea). Journal Eukaryotic Microbiology 56: 119-129. 
Fryer J \& M Mauel. 1997. The rickettsia: an emerging group of pathogens in fish. Emerging Infectious Diseases 3: 137144.

Fryer J \& R Hedrick. 2003. Piscirickettsia salmonis: a Gramnegative intracellular bacteria pathogen of fish. Journal of Fish Diseases 26: 251-262.

Fryer J, C Lannan, L Garcés, J Larenas \& P Smith. 1990. Isolation of a rickettsiales-like organism from diseased coho salmon (Oncorhynchus kisutch) in Chile. Fish Pathology 25: 107-114.

Garcés L, J Larenas, P Smith, S Sandino, C Lannan \& J Fryer. 1991. Infectivity of a rickettsia isolated from coho salmon Oncorhynchus kisutch. Diseases of Aquatic Organisms 11:93-97.

Gillespie J, K Williams, M Shukla, E Snyder, E Nordberg, S Ceraul, C Dharmanolla, D Rainey, J Soneja, J Shallom, N Vishnubhat, R Wattam, A Purkayastha, M Czar, O Crasta, J Setubal, A Azad \& B Sobral. 2008. Rickettsia phylogenomics: unwinding the intricacies of obligate intracellular life. PloS ONE 3: e2018. <http://dx.doi.org/ 10.1371/journal.pone.0002018>

Godoy M, J Troncoso, J Pontigol \& J Figueroa. 2013. Clinical and morphological spectrum of salmonid rickettsial septicemia (SRS). Journal of Fish Diseases 36: 587-591.

Gollas-Galván T, LA Avila-Villa, M Martínez-Porchas \& J Hernandez-Lopez. 2013. Rickettsia-like organisms from cultured aquatic organisms, with emphasis on necrotizing hepatopancreatitis bacterium affecting penaeid shrimp: an overview on an emergent concern. Reviews in Aquaculture 5: 1-14.

Gong W, X Xiong, Y Qi, J Jiao, C Duan \& B Wen. 2014. Identification of Novel Surface-Exposed Proteins of Rickettsia rickettsii by Affinity Purification and Proteomics. PLoS ONE 9(6): e100253. <doi:10.1371/journal. pone. 0100253>

Goodman R. 2010. Medical cell biology, 37 pp. Academic Press. San Diego.

Greub G \& D Raoult. 2003. History of the ADP/ATPTranslocase-Encoding Gene, a parasitism gene transferred from a Chlamydiales ancestor to plants 1 billion years ago. Environmental Microbiology 69: 5530-5535.

Harshbarger J, S Chang \& S Otto. 1977. Chlamydia (with phages), mycoplasmas and rickettsiae in Chesapeake Bay bivalves. Science 196: 666-668.

Hine P \& B Diggles. 2002. Prokaryote infections in the New Zealand scallops Pecten novaezelandiae and Chlamys delicatula. Diseases of Aquatic Organisms 50: 137-144.

Kanungo J, RM Empson \& H Rasmussen. 1999. Microinjection of an antibody to the $\mathrm{Ku}$ protein arrests development in sea urchin embryos. The Biological Bulletin 197: 341-347.
Karp G. 2013. Cell and molecular biology: Concepts and experiments, 864 pp. McGraw Hill, Ciudad de México.

Kleba B, T Clark, E Lutter, D Ellison \& T Hackstadt. 2010. Disruption of the Rickettsia rickettsii $\mathrm{Sca} 2$ autotransporter inhibits actin-based motility. Infection and Immunology 78: 2240-2247.

Küchler S, S Kehl \& K Dettner. 2009. Characterization and localization of Rickettsia sp. In water beetles of genus Deronectes (Coleoptera: Dytisciae). FEMS Microbiology Ecology 68: 201-211.

La M, P François, C Rovery, S Robineau, P Barbry, J Schrenzel, D Raoult \& P Renesto. 2007. Development of a method for recovering rickettsial RNA from infected cells to analyze gene expression profiling of obligate intracellular bacteria. Journal of Microbiological Methods 71: 292-297.

Larenas J, E Zamorano \& P Smith. 2005. Detección de Piscirickettsia salmonis en heces de salmón coho (Oncorhynchus kisutch) infectados por transmisión vertical. Electronic Monographs of Veterinary Pathology 2: 59-67.

Lodish B, K Matsudaira, S Krieger \& D Zipursky. 2005. Biología celular y molecular, 1054 pp. Editorial Médica Panamericana, México.

Luce F, A Maina, E Otiang, F Ade, S Omulo, E Ogola, L Ochieng, M Njenga \& A Richards. 2015. Isolation of Candidatus Rickettsia asemboensis from Ctenocephalides Fleas. Vector Borne Zoonotic Diseases 15: 268-277.

Martínez J. 2015. The roles of conserved outer-membrane proteins in SFG rickettsia pathogenesis, $10 \mathrm{pp}$. Louisiana State University A\&M Col Baton Rouge, Baton Rouge.

Martínez J \& P Cossart. 2004. Early signaling events involved in the entry of Rickettsia conorii into mammalian cells. Journal of Cell Science 117: 5097-5106.

Martínez J, S Seveau, E Veiga, S Matsuyama \& P Cossart. 2005. Component of DNA-dependent protein kinase, is a mammalian receptor for Rickettsia conorii. Cell 123: 10131023.

McQuiston J. 2016. Rickettsial (spotted \& typhus Fevers) and related infections (Anaplasmosis \& Ehrlichiosis). CDC Health Information for International Travel 2016. Oxford University Press, New York. <https://wwwnc.cdc.gov/travel/ yellowbook/2016/infectious-diseases-related-to-travel/ rickettsial-spotted-typhus-fevers-related-infectionsanaplasmosis-ehrlichiosis>

Morado J, A Sparks \& C O'Clair. 1988. A preliminary study of idiopathic lesions in the Dungeness crab, Cancer magister, from Rowan Bay, Alaska. Marine Environmental Research 26:311-318.

Morales-Covarrubias MS, L Tlahuel-Vargas, IE MartínezRodríguezE, R Lozano-Olvera \& JM Palaciosarriaga. 2011. NHP infection in Penaeus vannamei with florfenicol and oxytetracycline: a comparative experimental study. Revista Científica, FCV-LUZ 12(1): 72-80. 
Morales V \& J Cuellar. 2014. Guía técnica-patología e inmunología de camarones peneidos, 382 pp. OIRSA, Panamá.

Ngwamidiba M, G Blanc, H Ogata, D Raoult \& P Fournier. 2005. Phylogenetic study of Rickettsia species using sequences of the autotransporter protein-encoding gene sca2. Annals of the New York Academy of Sciences 1063: 94-99.

Noriea N, T Clark \& T Hackstadt. 2015. Targeted knockout of the Rickettsia rickettsii OmpA surface antigen does not diminish virulence in a mammalian model system. MBio 6: e00323-15.

Nunan M, T Poulos, S Navarro, M Redman \& V Lighter. 2010. Milky hemolymph syndrome (MHS) in spiny lobsters, penaeid shrimp and crabs. Diseases of Aquatic Organisms 91: 105-112.

Nunan LM, CR Pantoja, S Gomez-Jimenez \& DV Lightner. 2013. Candidatus Hepatobacter penaei, an intracellular pathogenic enteric bacterium in the hepatopancreas of the marine shrimp Penaeus vannamei (Crustacea: Decapoda). Applied and Environmental Microbiology 79: 1407-1409.

Oliver C, K Valenzuela, H Silva, R Haro, M Cortés, R Sandoval, J Pontigo, C Álvarez, J Figueroa, H Avendaño, J Troncoso \& A Yáñez. 2015. Effectiveness of egg yolk immunoglobulin against the intracellular salmonid pathogen Piscirickettsia salmonis. Journal of Applied Microbiology 199: 365-376.

Park H, L Hyuck, E Gouin, P Cossart \& T Izard. 2011. The rickettsia surface cell antigen 4 applies mimicry to bind to and activate vinculin. Journal of Biological Chemistry 286: 35096-35103.

Pereira S, S Bourrachot, I Cavalie, D Plaire, M Dutilleul, R Gilbin \& C Adam-Guillermin. 2011. Genotoxicity of acute and chronic gammairradiation on zebrafish cells and consequences for embryo development. Environmental Toxicology and Chemistry 30: 2831-2837.

Pulgar R, D Travisany, A Zuñiga, A Maass \& V Cambiazo. 2015. Complete genome sequence of Piscirickettsia salmonis LF-89 (ATCC VR-1361) a major pathogen of farmed salmonid fish. Journal of Biotechnology 212:30-31.

Rhee JS, RO Kim, BM Kim, HU Dahms \& JS Lee. 2012. Genomic organization of selected genes in the small monogonont rotifer, Brachionus koreanus. Gene 505: 108113.

Riley S, K Goh, T Hermanas, M Cardwell, Y Chan \& J Martinez. 2010. The Rickettsia conorii autotransporter protein Sca1 promotes adherence to nonphagocytic mammalian cells. Infection and Immunity 78: 1895-1904.
Roux V \& D Raoult. 2002. Phylogenetic analysis of members of the genus Rickettsia using the gene encoding the outermembrane protein $\mathrm{rOmpB}(\mathrm{ompB})$. International Journal of Systematic and Evolutionary Microbiology 50: 1449-1455.

Schmitz-Esser S, N Linka, A Collingro, CL Beier, HE Neuhaus, M Wagner \& M Horn. 2004. ATP/ADP translocases: a common feature of obligate intracellular amoebal symbionts related to Chlamydiae and Rickettsiae. Journal of Bacteriology 186: 683-691.

Stryer L, JM Berg \& JL Tymoczko. 2007. Biochemistry, pp. 529-530. W.H. Freeman, San Francisco.<https:// com mons.wikimedia.org/wiki/File:ATP ADP_Translocase_Mechanism.jpg>

Uchiyama T. 2012. Tropism and pathogenicity of rickettsiae. Frontiers in Microbiology 3(230), <http://dx.doi.org/10.3389>

Valbuena G. 2010. Patogénesis de las infecciones producidas por rickettsias en las Americas. Revista MVZ Córdoba 15: 2004-2006.

Wang W \& Z Gu. 2002. Rickettsia-like organism associated with tremor disease and mortality of the Chinese mitten crab Eriocheir sinensis. Diseases of Aquatic Organisms 48: 149153.

Wang Y, G Kwon, H Li \& G LeBlanc. 2011. Tributyltin synergizes with 20-hydroxyecdysone to produce endocrine toxicity. Toxicological Sciences 123: 71-79.

Whitworth T, V Popov, X Yu, D Walker \& D Bouyer. 2005. Expression of the Rickettsia prowazekii pld or tlyC gene in Salmonella enterica serovar Typhimurium mediates phagosomal escape. Infection and Immunity 73: 6668-6673.

Wu X. 2003. Advances in the research of marine-cultured animal diseases in China. In: Lee C-S \& A Ventura (eds). Status of aquaculture in China, pp. 29-54.The Oceanic Institute, Hawaii.

Yang A, A Narechania \& E Kim. 2016. Rickettsial endosymbiont in the 'earlydiverging' streptophyte green alga Mesostigma viride. Journal of Phycology 52(2): 219-229.

Zewen Z, X Ting, H Zhongyang, W Xinzhong, W Liuji, M Qingguo \& H Jiaqing. 2012. Rickettsia-like organism infection associated with mass mortalities of blood clam, Tegillarca granosa, in the Yueqing Bay in China. Acta Oceanológica Sinica 31: 106-115.

Zhu B \& X Wu. 2008. Identication of outer membrane protein ompR from rickettsia-like organism and induction of immune response in Crassostrea ariakensis. Molecular Immunology 45:3198-3204. 